\title{
REAL-TIME DENSITY MEASUREMENT USING COMPACTOR VIBRATORY RESPONSES
}

\author{
by
}

\author{
R. Edward Minchin Jr., PE \\ Instructor, The Pennsylvania State University \\ H. Randolph Thomas Jr., PhD, PE \\ Professor, The Pennsylvania State University
}

\begin{abstract}
This paper deals with research into the possibility of using the vibratory responses of a soil/asphalt compactor to determine the relative density of the material being compacted.
\end{abstract}

\section{INTRODUCTION}

State Highway Agencies, as well as federal, county, and municipal agencies now require that contractors perform much of their own Quality Control. At the same time, there is a shortage of qualified inspectors and equipment operators.

One of the areas that automation can practically be of great and immediate benefit is in the area of Quality Control in general and in the area of embankment, base, and pavement compaction, in particular. Perhaps the most costly item for an owner or contractor to verify is the density of the asphalt mat, base, or embankment. Nuclear density gauges have served the industry well, but are virtually unchanged from those used fifteen years ago.

There are many specifications and procedures to assure that the specified density is achieved. However, many specifications only require one density reading per 1000 linear feet of lane width. It is prohibitively expensive to retain the manpower necessary to take nuclear density readings for the entire length of the pavement. Thus there might well be many areas where the density is less than is specified, and these areas remain unknown to the owner and contractor until the asphalt in these areas begins breaking down in some way.

\section{The History of Onboard Density Meters}

Onboard density meters have traditionally been used for projects that are very time-sensitive, or where the compactor operator is the chief source of quality control [1].
These meters have been popular in Europe for years. European construction companies pride themselves on a smaller construction crew with highly skilled craftsmen and equipment operators. Their production is much smaller, but so is their overhead.

Getting a better job the first time and not having to redo work is very appealing to a contractor. The relatively new and very vigorous push to shorten project duration times is sure to heighten interest in North America for this "compaction-on-the-fly" technology.

\section{State of the Art}

Current technology is such that the densities given by onboard compaction meters are relative densities - not true densities. Only nuclear density gauges give true densities. Even so, the meters are quite popular in Europe because they speed up the compaction process. The amount of benefit that a company derives from a unit is proportional to the skill of the operator. At this point, the meters are just another tool to help a knowledgeable construction crew make quicker and better decisions in the course of their work.

As for the units themselves, the most popular technology currently employed in Europe is to mount a vibration sensor in the roller wheel which detects the motion of the drum and relays the signal to the meter's processor. Increasing reaction forces correspond to growing compaction, and the results are displayed as a numerical value or graphically, according to the system chosen [1].

The present systems used in Europe are priced from $\$ 10,000$ to $\$ 15,000$ and can not accurately indicate the specific point on the mat from which a density reading was rendered [1]. Still, a contractor involved in a high volume of time-sensitive work can make the investment pay. American contractors, however, can not, at this time, justify this added cost on their equipment. The competition is too keen, and project owner and contractor interest is still too low. 


\section{THE OBJECTIVE}

The goal of this research is to establish a relationship between the relative density of a material and the level of vibratory excitement of the acoustic waves generated by a compactor as it is compacting that material. The system, in its entirety, will offer the features of real-time documentation of location, density readings, and mat temperature. However, discussion of this total system and how the Onboard Density Meter is integrated into the system is beyond the scope of this paper.

\section{THE SYSTEM}

The Penn State Compaction System bases relative density on the hypothesis that the equipment vibratory response is related to the stiffness of the material on which the compactor is operating. That is, more dense material causes the compactor to vibrate with greater acceleration at a certain frequency or frequencies on smooth-wheel compactors, and sheepsfoot-type equipment

\section{Asphalt Density Measurements using Vibration Principles}

The assertion that asphalt density is related to equipment vibration responses is based on the mechanical model in Figure 1. A compactor has intrinsic vibrations from the engine, propulsion system, and vibrators in the rollers which shake the equipment up and down as it moves. The vibration of the equipment is influenced by a number of other factors as described below. this too is not of great consequence since the compaction operations is typically done at nearly the same speed. Thus, the consequence is that the vibration forces $f(t)$ remain constant (see Figure 1), and mass and speed are not major factors in this analysis.

The acceleration $\mathrm{a}(\mathrm{t})$ of the compactor vibrations depends on the ground stiffiness $\mathrm{K}$ and damping $\mathrm{R}$. At very low frequencies (say below $25 \mathrm{~Hz}$ ), the stiffness $\mathrm{K}$ is the dominant physical factor affecting $a(t)$, so that the damping $R$ can be ignored. Thus, with constant $f(t)$ and $M$ and negligible $\mathrm{R}$, the acceleration $\mathrm{a}(\mathrm{t})$ is proportional to the stiffness $\mathrm{K}$ of the medium being compacted. Mathematically,

\section{Acceleration $\mathrm{a}(\mathrm{t}) \approx$ Stiffness $\mathrm{K}$}

As materials beneath the compactor becomes more dense, the reduction of air voids adds structure to the material. This results in higher densities and greater overall ground stiffness. This relationship is expressed as follows:

\section{Stiffness $\mathrm{K} \approx$ Density or Compaction}

It follows that:

\section{Acceleration $\mathrm{a}(\mathrm{t}) \approx$ Density or Compaction}

Increased stiffness excites the specific excitation vibrations already being produced by the compactor. As the equipment moves over the compacted material, the vibration forces result in higher acceleration amplitudes compared to when the equipment passes over softer material. Thus, based

\section{Mechanical Model}

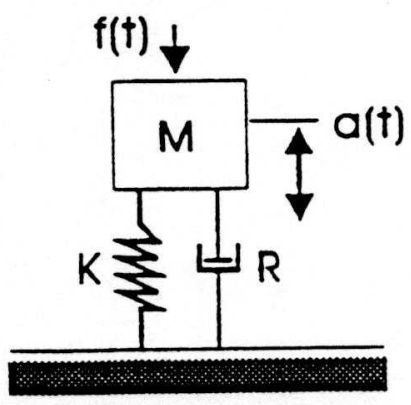

Roller

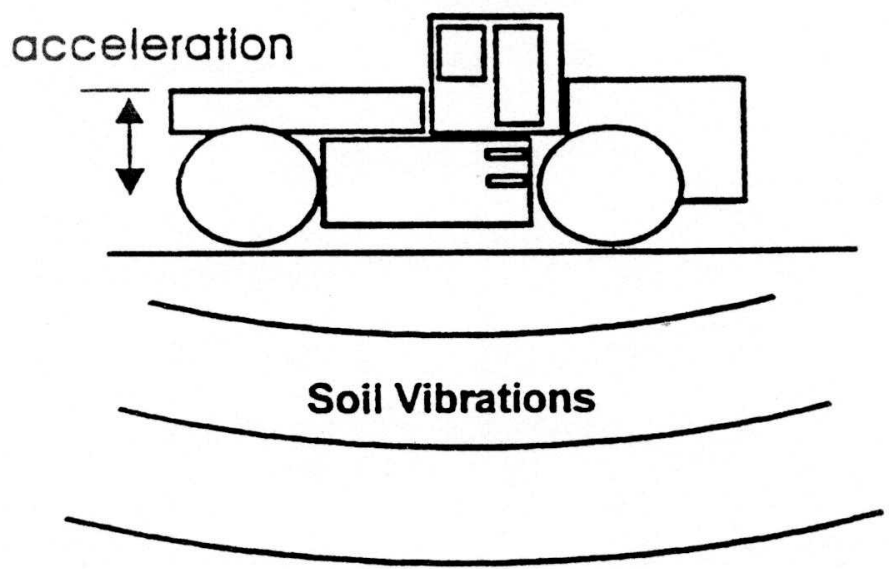

Figure 1. Mechanical model for roller vibration response of soil.

The mass of the roller or compactor $M$ influences the vibration forces $f(t)$, but the mass remains constant throughout use. Equipment speed also influences $f(t)$, but on the simplistic model in Figure 1, the following expectations have been observed in field evaluations on asphalt mats: 
- the compactor produces low frequencies (less than $25 \mathrm{~Hz}$ )

- as the asphalt stiffens, the amplitude of the acceleration $a(t)$ also increases

- increases in acceleration $\mathrm{a}(\mathrm{t})$ are proportional to increases in compaction

- higher compactor speeds cause slight increases in peak frequencies, but has little affect on accelerations $\mathrm{a}(\mathrm{t})$

\section{Relationship Between Density and Acceleration}

The equipment vibration data is analyzed for each pass on a test strip to determine the power spectrum or frequency domain. Using these data and the other information, other analyses and correlations are performed as detailed below.

For each test strip, the relationship between the number of passes and the equipment vibratory response measured in $\mathrm{dBs}$ is performed. The consistency between test strips is noted and peculiarities are documented. Project-toproject differences are also of particular interest.

For each test strip, the vibratory responses are measured for each pass for the fundamental frequency and the first five harmonic frequencies. For each test strip, plots of changes in acceleration are developed. Based on these plots, the most responsive frequencies are identified. These are the candidate frequencies. Non responsive frequencies are eliminated from further consideration. If equipment models change, the analysis is repeated.

Plots of the equipment vibratory response measured in $\mathrm{dBs}$ for the candidate frequencies are correlated to the actual density values measured in pcf using a nuclear density gauge. Frequencies that are most responsive to density are retained for further analysis.

\section{THE TECHNOLOGY}

The Automated Compaction Meter, is comprised of several key components. The process all starts with an accelerometer. The accelerometer is a transducer which contains a quartz crystal mass. The movement of this mass produces voltage proportional to the acceleration of the mass. This produces a piezo-electric effect in which the mass and base move with the same acceleration when the frequency of movement is below natural frequency. Thus, the accelerometer 'measures' the acceleration of the vibratory movement of the compactor.

When the accelerometer is properly attached to a compactor, the acceleration of the mass should mirror the acceleration of the compactor. The measurements are then processed through a spectrum analyzer VIA a Digital Audio Tape (DAT) recorder, and needed graphs are generated.

\section{THE PROCEDURE}

Data utilized in this paper are field data taken according to a precise data acquisition plan. Materials over which the system has been tested include non-homogeneous trash in a landfill, embankment, rock base material, and various asphalt courses.

The focus of this paper is data taken from a compactor operated by Glenn O. Hawbaker, Inc. on an Interstate 99 project in Bellfonte, PA. The material and layer being compacted was a rock base course. Readings were taken from multiple (six) passes over the same 200-ft. test strip.

Analysis consists of several steps. One of these steps is the generation of a power spectrum at the point where nuclear density readings are taken. The data is thus transformed to the frequency domain. Once accomplished, this allows the researcher to ascertain which frequency is most excited by an increase in density. The researcher can then relate density to the level of acceleration/vibration. The data taken from the field sites has been very encouraging.

The accelerometer used to date has been a micromachined accelerometer. The readings taken by the accelerometer are transmitted to a DAT recorder. The current DAT recorder is connected by wire to the accelerometer. Eventually, a wireless system must be utilized.

The DAT recorder, is now hooked up to a Spectrum Analyzer, in this case an HP 3567A. The DAT tape is then played into the analyzer. The analyzer is interfaced through an HP Instrument Bus to a computer. It is the analyzer, however, which performs all the work, through Fast Fourier Transform (FFT) analysis.

\section{THE RESULTS}

For the purposes of this paper, only the data collected from a Caterpillar CS 534 Compactor with dual smooth-wheel vibratory rollers will be discussed. This machine was instrumented on the project mentioned earlier.

The six passes which were recorded were equally divided as to direction of the compactor. The even-numbered passes were all in one direction, the odd-numbered were in the other. Figure 2 shows the Power Spectrums for the oddnumbered passes plotted together. A very definite pattern is discernable. Power Spectrums were then generated for each of the six passes (Figure 3 shows the Power Spectrum generated for pass number six).

Keeping the passes separated by direction, the frequencies which appeared as "spikes" in the power spectrums were further analyzed. The values of these frequencies were taken from the power spectrums and plotted (Figure 4 shows these plotted values for the even-numbered passes). It should be pointed out that the power spectrum is plotted on a logarithmic scale, so the actual differences 


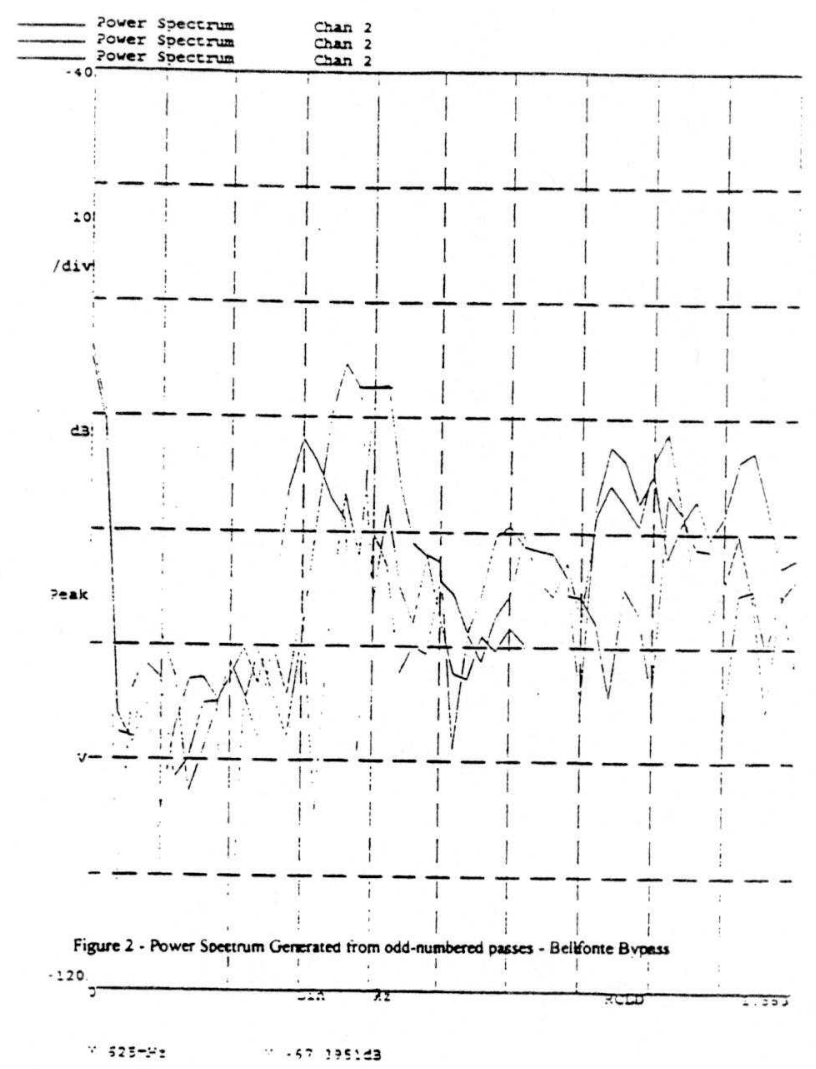

Figure 2. Power spectrum generated from odd-numbered passes - Bellefonte Bypass.

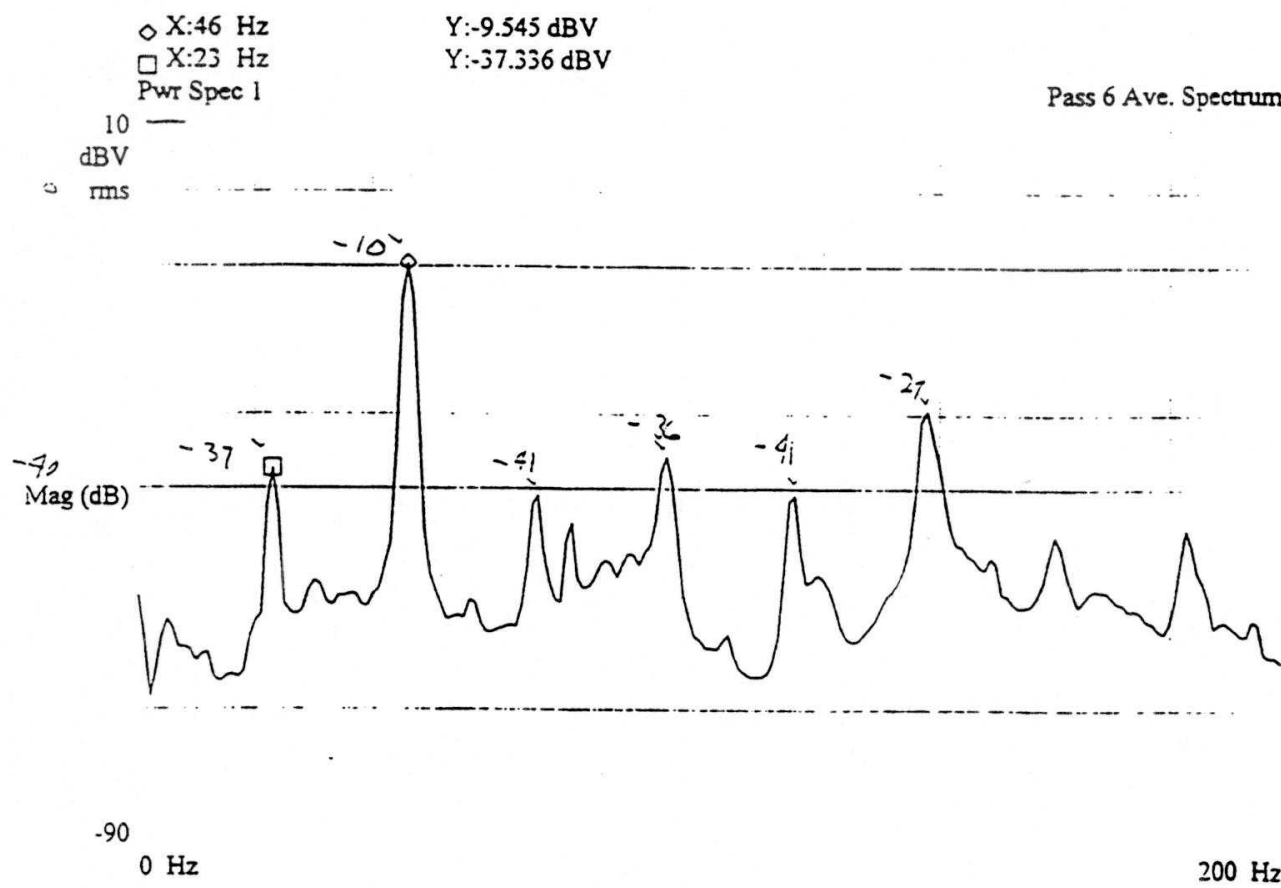

Figure 3. Power spectrum generated from Pass No. 6 - Bellefonte Bypass. 
between these "spikes" and the lower-level noise is actually larger than it appears in Figures Numbered Two and Three.

The frequencies which showed the most promise were the fundamental frequency and the first harmonic frequency. These frequencies were further studied. The reason these two particular frequencies seem to show promise was that the fundamental frequency and the second harmonic frequency each rendered plots in which the acceleration increases with each successive pass.

\section{CONCLUSIONS}

It is believed that only one frequency is the one emitted by the wheel impacting the material. The two frequencies just discussed seem to be the best candidates. Therefor, these two frequencies were studied further still. Density readings were obtained for the material immediately following each of the six passes. The density readings were taken at the same preordained spot on the roadway at which the acceleration readings were taken. The acceleration readings were then plotted against densities at the same spot in the road (Figure 5 shows this Density v Acceleration plot for the two frequencies deemed worthy of further consideration). The plot for each of the six passes showed an increase in acceleration (measured in decibels) which corresponded to an increase in density.

This is quite significant as it seems to confirm the original hypothesis of the research. While very encouraging, this is a single sample, and much more research must be done before any firm conclusions can be drawn.

\section{REFERENCES}

1. L. Geistlinger, "Onboard Compaction Meters Make Inroads Into U.S. Market", Roads and Bridges, August, 1996 
Fundamental

$\ldots \ldots \ldots . . .2$ 2nd Harmonic

- - 4th Harmonic
- 1ar Harmonic

- - 3rd Harmonic

-... 5th Harmonic

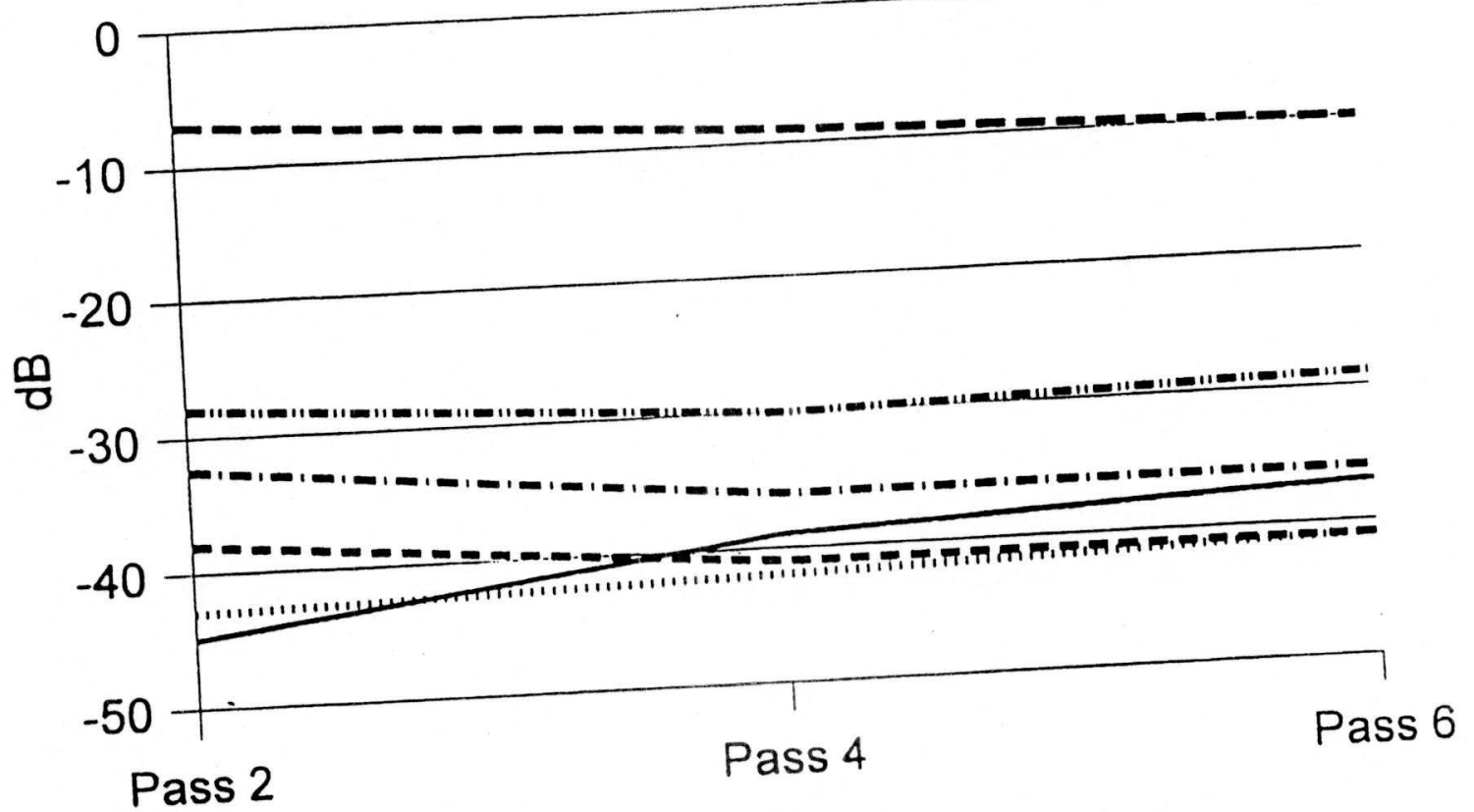

Figure 4. Frequencies plotted from even-numbered power spectrums.

Density vs. $d B$

2nd, 4th, \& 6th Passes

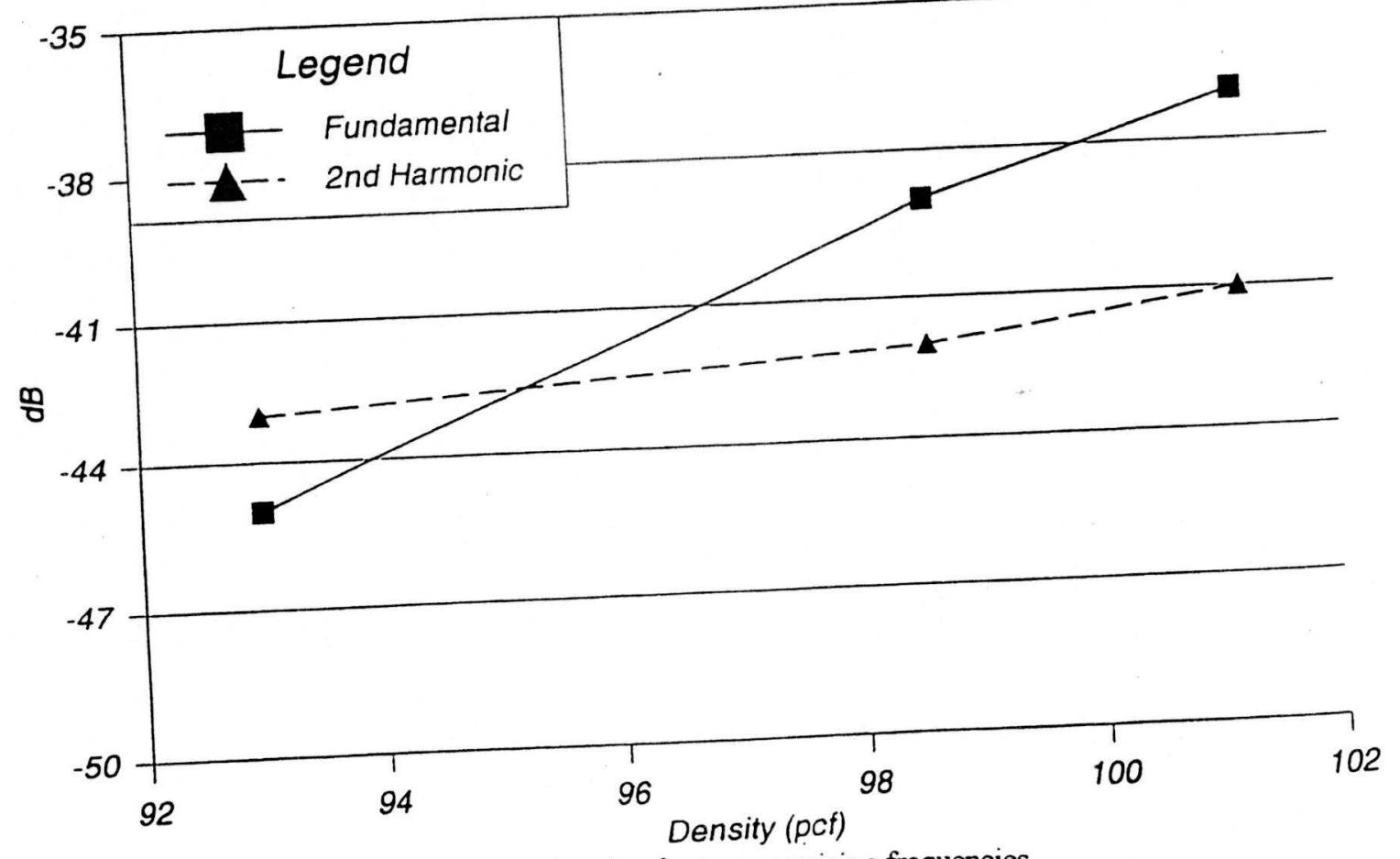

Figure 5. Density v. acceleration for two promising frequencies. 